\title{
Understanding the Solid-State Hydration Behaviour of a Common Amino Acid: Identification, Structural Characterization and Hydration/Dehydration Processes of New Hydrate Phases of L-Lysine
}

\author{
P. Andrew Williams,,${ }^{a}$ Colan E. Hughes, ${ }^{a}$ Jean Martin, ${ }^{a}$ Emilie Courvoisier,${ }^{a}$ Asma B. M. Buanz, ${ }^{b}$ \\ Simon Gaisford ${ }^{b}$ and Kenneth D. M. Harris ${ }^{* a}$ \\ ${ }^{a}$ School of Chemistry, Cardiff University, Park Place, Cardiff CF10 3AT, Wales, U.K. \\ ${ }^{b}$ School of Pharmacy, University College London, 29-39 Brunswick Square, London WC1N 1AX, \\ U.K.
}

* Author for correspondence: HarrisKDM@ cardiff.ac.uk

\begin{abstract}
The crystal structure of L-lysine was reported very recently and represented the final member of the set of 20 directly-encoded proteinogenic amino acids to have a crystal structure of the enantiomerically pure form determined. Under ambient conditions, L-lysine has a strong propensity to undergo hydration by incorporation of water into the crystal structure and dehydration under rigorously dry conditions is required to obtain anhydrous L-lysine. In the present paper, we explore the hydration of L-lysine, which has led to the identification and characterization of a hemihydrate phase and a monohydrate phase. As the hydration processes give rise to microcrystalline powder samples of the hydrate phases, powder X-ray diffraction has been exploited for crystal structure determination of both L-lysine hemihydrate and L-lysine monohydrate. The conditions for interconversion between anhydrous L-lysine and the two hydrate phases has been investigated by dynamic vapour sorption measurements, leading to an understanding of the hydration/dehydration behaviour in this system.
\end{abstract}

\section{Keywords}

powder X-ray diffraction, dynamic vapour sorption, crystal forms, hydration/dehydration processes, solid-state transformations 


\section{Introduction}

Recently, the crystal structure of L-lysine (Fig. 1) was determined directly from powder X-ray diffraction (XRD) data, ${ }^{1}$ representing the final member of the set of 20 genetically encoded proteinogenic amino acids to have a crystal structure determined for the enantiomerically pure form. ${ }^{2}$ However, L-lysine is known to undergo hydration very readily under normal atmospheric conditions. Indeed, the powder sample of L-lysine used for structure determination from powder XRD data ${ }^{1}$ originated as a hydrate phase, which was then subjected to a rigorous dehydration procedure prior to recording the powder XRD data. The observation that L-lysine undergoes facile hydration under ambient atmospheric conditions prompted us to investigate the hydration/dehydration behaviour in more detail. The results of these investigations are reported here, including determination of the structural properties of two new hydrate phases directly from powder XRD data.

Among recent work on the solid phases of amino acids, the existence of two distinct hydrate phases of L-phenylalanine (in one case a non-stoichiometric hydrate ranging in composition from a quarter-hydrate to a hemihydrate and in the other case a monohydrate) and a new polymorph of L-phenylalanine (polymorph II) has been reported. ${ }^{3}$ The fact that isolation of polymorph II of L-phenylalanine requires rigorously anhydrous conditions is reminiscent of the situation encountered in preparing the anhydrous form of L-lysine described above. Anhydrous conditions were also required to prepare a suitable powder sample of the anhydrous form of L-arginine, to allow crystal structure determination to be carried out from powder XRD data. ${ }^{4}$

Prior to the present work, crystal structures of hydrate phases have been reported for only seven of the 20 directly encoded proteinogenic amino acids. In one case, a hydrate crystal structure is known only for the racemic form (DL-glutamic $\operatorname{acid}^{5}$ ), in three cases a hydrate crystal structure is known only for the enantiomerically pure form (L-asparagine, ${ }^{6} \mathrm{~L}$-aspartic acid ${ }^{7}$ and L-phenylalanine ${ }^{3}$ ), and in three cases hydrate crystal structures are known for both the racemic and enantiomerically pure forms (arginine ${ }^{8-9}$ proline $^{10-11}$ and serine ${ }^{12-13}$ ).

We report here the discovery of two hydrate phases of L-lysine. As discussed below, under ambient conditions of low to moderate relative humidity $(\mathrm{RH})$, L-lysine is found as a hemihydrate, and anhydrous L-lysine exists only under very dry conditions. When exposed to an atmosphere of 
higher humidity ( $c a .50 \% \mathrm{RH}$ or higher), L-lysine is found to form a monohydrate phase prior to deliquescence. In this paper, we report the crystal structures of both L-lysine hemihydrate and L-lysine monohydrate, which have been determined directly from powder XRD data. The use of powder XRD data for structure determination is essential in these cases due to the difficulty of preparing single crystals of suitable size and quality for single-crystal XRD studies.

Although the task of determining crystal structures from powder XRD data is significantly more challenging than from single-crystal XRD data, advances over the past 20 years or so in techniques for analysis of powder XRD data ${ }^{14-20}$ are such that the structural properties of organic materials of moderate complexity can now be determined relatively routinely from powder XRD data. In the case of molecular solids, the direct-space strategy ${ }^{15}$ for structure solution from powder XRD data has created considerable opportunities for the direct structural characterization of materials prepared by processes that intrinsically generate microcrystalline powders as the product phase, including those prepared by solid-state hydration and dehydration processes. ${ }^{1,3-4,21-22}$

\section{Experimental}

In the present work, powder XRD data were recorded on a Bruker D8 diffractometer (Ge monochromated $\mathrm{CuK} \alpha_{1}$ radiation) operating in transmission mode. The sample of L-lysine was purchased from Sigma-Aldrich (purity $\geq 98 \%$ ). Under ambient conditions, the powder XRD pattern of this sample is different from that of anhydrous L-lysine. ${ }^{1}$ On the basis of dynamic vapour sorption (DVS) measurements, the purchased material was established to be L-lysine hemihydrate.

L-Lysine monohydrate was prepared by exposing L-lysine hemihydrate to an atmosphere of high RH. Specifically, a powder sample of L-lysine hemihydrate in a small glass beaker was placed in a Petri dish filled with water; the Petri dish and small beaker were covered with a larger inverted beaker to provide an enclosed environment. After several hours, deliquescence was found to begin. The portion of the sample that had not yet undergone deliquescence was removed and powder XRD data were recorded. The powder XRD pattern was different from those of anhydrous L-lysine and L-lysine hemihydrate, and the material was established to be a monohydrate of L-lysine from DVS measurements (this assignment was confirmed subsequently by structure determination from powder XRD data). 
To record high-quality powder XRD data suitable for crystal structure determination, the powder sample of L-lysine hemihydrate was mixed with amorphous starch (to reduce the effects of preferred orientation) and packed into glass capillaries (to maintain an atmosphere of constant $\mathrm{RH}$ ) prior to flame sealing. In the case of L-lysine monohydrate, the powder sample was contained between two pieces of tape in a foil-type sample holder. Powder XRD data were recorded for L-lysine hemihydrate and L-lysine monohydrate over the $2 \theta$ range $4-70^{\circ}$, with step size $0.017^{\circ}$. For L-lysine hemihydrate, the step time was $15 \mathrm{~s}$ and the total data collection time was $16 \mathrm{hrs} 52 \mathrm{~min}$. For L-lysine monohydrate, the step time was $14.2 \mathrm{~s}$ and the total data collection time was $15 \mathrm{hrs} 58 \mathrm{~min}$.

DVS experiments were carried out at $26.2 \pm 0.1{ }^{\circ} \mathrm{C}$ on a DVS Advantage 1 instrument (Surface Measurement Systems Ltd., London, U.K.). Samples were placed in glass pans and were kept initially at $0 \% \mathrm{RH}$ for $2 \mathrm{hrs}$. The target $\mathrm{RH}$ was then increased to either $25 \%$ or $95 \%$ in a step of $5 \%$ every hour and then decreased to $0 \%$ (in a step of $-5 \%$ every hour). The partial pressure of water in the sample environment was controlled by mixing water-saturated nitrogen and dry nitrogen (oxygenfree) gases, facilitated using an electronic mass flow controller. The mass of the sample was recorded as a function of RH using a fitted microbalance.

\section{Results}

\section{Dynamic Vapour Sorption}

The hydration/dehydration behaviour of L-lysine was investigated using DVS measurements starting from a sample of anhydrous L-lysine which had been dried under vacuum in the presence of $\mathrm{P}_{2} \mathrm{O}_{5}$ for three days. In the first DVS measurement (Fig. 2), the RH was increased from $0 \%$ to $95 \%$ and then decreased to $0 \%$ in increments of $5 \% \mathrm{RH}$, with $1 \mathrm{hr}$ at each target value of $\mathrm{RH}$. Uptake of water was found to occur at $c a .5 \% \mathrm{RH}$, with a mass increase corresponding to one half equivalent of water. On further increasing $\mathrm{RH}$, the sample mass remained constant until ca. 55\% RH, after which the mass of the sample increased substantially and in a continuous manner as RH was increased to $95 \%$. This substantial increase in mass is attributed to deliquescence. The $\mathrm{RH}$ was then decreased and the mass of the sample was observed to decrease between $c a .85 \% \mathrm{RH}$ and $40 \% \mathrm{RH}$. The mass then remained constant on further decreasing RH to $0 \%$. The final mass was considerably greater than the starting 
mass (and considerably higher than the mass predicted for a monohydrate phase), suggesting that an irreversible hydration event had occurred. The recovered sample was a transparent glassy substance.

A second DVS experiment (Fig. 3) was carried out over a narrower range of RH, starting with a sample of anhydrous L-lysine (dried under vacuum in the presence of $\mathrm{P}_{2} \mathrm{O}_{5}$ ) at $0 \%$. The $\mathrm{RH}$ was then increased to $25 \%$ and subsequently decreased to $0 \%$ in target increments of $5 \% \mathrm{RH}$ with $1 \mathrm{hr}$ at each target value of $\mathrm{RH}$. At values of RH between $\mathrm{ca} .2 \%$ and $13 \%$, the mass of the sample was observed to increase, corresponding to uptake of one half equivalent of water and leading to the assignment of the resultant phase as L-lysine hemihydrate. No further increase in mass was observed on increasing $\mathrm{RH}$ to $25 \%$. On subsequently decreasing $\mathrm{RH}$, a decrease in mass corresponding to one half equivalent of water was observed between $c a .2 \%$ and $0 \% \mathrm{RH}$, indicating that the transformation between anhydrous L-lysine and L-lysine hemihydrate is reversible (although with hysteresis).

Alternative plots showing the results of the DVS experiments with the data plotted as percentage mass versus RH are included in Supporting Information (Figs. S1 and S2).

\section{Structure Determination from Powder XRD Data}

The powder XRD patterns of L-lysine hemihydrate and L-lysine monohydrate were indexed using, respectively, the $\mathrm{KOHL}^{23}$ and $\mathrm{ITO}^{24}$ algorithms in the $\mathrm{CRYSFIRE}^{25}$ indexing suite, giving a unit cell with monoclinic metric symmetry for L-lysine hemihydrate $(a=9.54 \AA, b=5.22 \AA, c=17.61 \AA$, $\beta=101.1^{\circ} ; \quad V=860.6 \AA^{3}$ ) and a unit cell with orthorhombic metric symmetry for L-lysine monohydrate $\left(a=5.94 \AA, b=20.61 \AA, c=6.95 \AA ; V=850.3 \AA^{3}\right)$. Profile fitting and unit cell refinement were carried out using the Le Bail procedure ${ }^{26}$ implemented in the program GSAS. ${ }^{27}$ On the basis of systematic absences, the space group of the hemihydrate was assigned as $C 2$ and the space group of the monohydrate was assigned as $P 2{ }_{1} 2{ }_{1} 2{ }_{1}$. In each case, good quality Le Bail fits were achieved (L-lysine hemihydrate, $R_{\mathrm{wp}}=2.88 \%, R_{\mathrm{p}}=2.17 \%$, Fig. 4a; L-lysine monohydrate, $R_{\mathrm{wp}}=2.57 \%, R_{\mathrm{p}}=1.94 \%$, Fig. 5a). Given the unit cell volumes and density considerations, it was established that the asymmetric unit for the hemihydrate comprises one molecule of L-lysine and one half molecule of water (located on the 2-fold rotation axis in space group $C 2$ ) and the asymmetric unit for the monohydrate comprises one molecule of L-lysine and one molecule of water. 
Structure solution was carried out from the powder XRD data using the direct-space genetic algorithm (GA) technique ${ }^{28-32}$ in the program EAGER ${ }^{33-39}$ In the structure-solution calculations, the L-lysine molecules were defined as zwitterions, with the amine group in the head group (rather than the amine group at the end of the side chain) assigned as the protonated base, as found in the crystal structure of anhydrous L-lysine. ${ }^{1}$

In the structure-solution calculation for the hemihydrate, the L-lysine molecule (including hydrogen atoms) was defined by 10 structural variables: 2 positional variables (for space group $C 2$, the origin can be assigned arbitrarily along the $b$-axis, allowing the positional variable along this axis to be fixed for one molecule), 3 orientational variables and 5 torsional variables. The water molecule (with half occupancy) was included in the structure-solution calculation as an oxygen atom fixed on the 2 -fold rotation axis [i.e., with coordinates $(0, y, 1 / 2)]$; the hydrogen atoms of the water molecule were not included in the calculation, and thus the water molecule was defined by 1 positional variable.

In the structure-solution calculation for L-lysine monohydrate, the L-lysine molecule was defined by 11 structural variables ( 3 positional variables, 3 orientational variables and 5 torsional variables). The water molecule (including hydrogen atoms) was defined by 6 structural variables ( 3 positional variables and 3 orientational variables).

For both L-lysine hemihydrate and L-lysine monohydrate, 16 independent GA structuresolution calculations were carried out. In all calculations, the population comprised 400 trial structures and the calculation was run for 500 generations, with 40 mating operations and 200 mutation operations carried out per generation. In each case, the 16 independent calculations resulted in the same structure of highest quality (i.e., lowest $R_{\mathrm{wp}}$ ).

The best trial structure obtained in the structure-solution calculations was used as the starting point for Rietveld refinement ${ }^{40}$ using the program GSAS. ${ }^{27}$ Standard restraints were applied to bond lengths ${ }^{41}$ and bond angles, and planar restraints were applied to the carboxylate groups. For each molecule (L-lysine or water), a common isotropic displacement parameter was refined for the nonhydrogen atoms. The isotropic displacement parameter for hydrogen atoms was set as 1.2 times the refined isotropic displacement parameter for the non-hydrogen atoms in the same molecule. 
Intermolecular distance restraints were used to allow the hydrogen-bond donors to be oriented in a manner corresponding to optimal hydrogen-bonding geometry.

In the final Rietveld refinement for the hemihydrate, disorder in the hydrogen-bonding scheme involving the side-chain amine groups of L-lysine and the water molecules was introduced into the structural model. An ordered model was judged to be insufficient to describe the crystal structure as there are multiple possible hydrogen bonding arrangements involving the side-chain amine groups and water molecules that are considered to be essentially equally favourable in terms of geometric criteria for the hydrogen-bond distances and angles. Specifically, the hydrogen atoms of the amine group and the hydrogen atoms of the water molecule were disordered over 6 sites with occupancies fixed at $1 / 3$ (corresponding to a total of 2 hydrogen atoms in the amine group and 2 hydrogen atoms in the water molecule).

For L-lysine monohydrate, a correction for preferred orientation was included in the Rietveld refinement using the March-Dollase function. ${ }^{42-43}$

For both the hemihydrate and the monohydrate, the final Rietveld refinement gave a good fit to the experimental powder XRD data (L-lysine hemihydrate, $R_{\mathrm{wp}}=3.13 \%, R_{\mathrm{p}}=2.35 \%$; L-lysine monohydrate, $R_{\mathrm{wp}}=2.88 \%, R_{\mathrm{p}}=2.13 \%$ ), and the final refined parameters were as follows: L-lysine hemihydrate, $\quad C 2, \quad a=9.53090(27) \AA, \quad b=5.21728(17) \AA, \quad c=17.6011(8) \AA, \quad \beta=101.078(4)^{\circ}$, $V=858.91(6) \AA^{3}$ (Fig. 4b; $2 \theta$ range, $4^{\circ}-70^{\circ} ; 3866$ profile points; 117 refined variables); L-lysine monohydrate, $P 2{ }_{1} 2{ }_{1} 2_{1}, a=5.93493(10) \AA, b=6.94133(11) \AA, c=20.5754(6) \AA, V=847.63(4) \AA^{3}$ (Fig. $5 \mathrm{~b} ; 2 \theta$ range, $4^{\circ}-70^{\circ} ; 3867$ profile points; 102 refined variables).

Plots showing the results from the Le Bail fitting and the final Rietveld refinement with the background intensity included in the data are shown in Supporting Information for L-lysine hemihydrate (Fig. S3) and L-lysine monohydrate (Fig. S4).

\section{Discussion}

The crystal structure of L-lysine hemihydrate (Fig. 6) may be described as a layered structure containing two different regions of hydrogen bonding (in this aspect, the structure resembles that of anhydrous L-lysine ${ }^{1}$ ). In one region, the hydrogen bonding involves the ammonium and carboxylate 
groups of the amino acid head-groups of the L-lysine molecules. In the other region, the hydrogen bonding involves the water molecules and the amine groups at the termini of the side-chains of the Llysine molecules. In anhydrous L-lysine, the hydrogen bonding in the latter region solely involves amine groups to form chains of $\mathrm{N}-\mathrm{H} \cdots \mathrm{N}$ hydrogen bonds. In the hemihydrate, on the other hand, the water molecule is incorporated into the hydrogen bonding arrangement involving the side-chain amine groups; each amine group is surrounded by three other amine groups and three water molecules, each of which acts as both a hydrogen-bond donor and a hydrogen-bond acceptor. Similarly, each water molecule is surrounded by six amine groups, each of which acts as both a hydrogen-bond donor and a hydrogen-bond acceptor. As shown in Fig. 7, an extended network of N$\mathrm{H} \cdots \mathrm{N}$ hydrogen bonds is formed parallel to the $a b$-plane, constructed from 6-membered rings (highlighted in cyan) in a chair-type arrangement, with the nitrogen atoms of amine groups at the apices. Two further networks (highlighted in magenta and yellow) of edge-sharing 6-membered rings, also in chair-type arrangements, are interlinked with the first network. The latter two networks involve $\mathrm{N} \cdots \mathrm{H}-\mathrm{O}$ and $\mathrm{N}-\mathrm{H} \cdots \mathrm{O}$ hydrogen bonds, with oxygen atoms of water molecules and nitrogen atoms of amine groups alternating at the apices. For each amine group and each water molecule, there are six possible positions for the hydrogen atoms to serve as donors in the formation of geometrically reasonable hydrogen bonds to neighbouring groups. For this reason, a disordered model for the positions of these hydrogen atoms was used in the final Rietveld refinement calculations, with each hydrogen atom disordered with equal probability among six possible positions.

In the crystal structure of anhydrous L-lysine, there are two independent molecules in the asymmetric unit with appreciably different conformations, whereas the crystal structure of the hemihydrate has only one independent molecule of L-lysine. The water molecule in L-lysine hemihydrate is located on a 2 -fold rotation axis, resulting in a 2:1 ratio of L-lysine to water.

The hydrogen bonding between the amino acid head-groups in L-lysine hemihydrate (Fig. 8) can be described using the classification system proposed by Görbitz et al. ${ }^{44}$ Specifically, the structure contains sheets of type $\mathrm{L} x$, in contrast to the sheets of type $\mathrm{L} 2$ found in anhydrous L-lysine. We note that L-lysine hemihydrate is actually unique among the hydrates of amino acids as it is the first case of a hydrate phase of an amino acid that can be classified by this system. All previously reported crystal structures of hydrate phases either do not form the requisite layered arrangement or else 
contain water molecules that participate in the hydrogen bonding arrangement involving the amino acid head-groups. For the latter reason, the crystal structure of L-lysine monohydrate reported here cannot be classified using this system.

While the crystal structures of anhydrous L-lysine and L-lysine hemihydrate share several similarities (see Fig. 9), the crystal structure of L-lysine monohydrate (Fig. 10) differs significantly. The structure of the monohydrate contains only one hydrogen-bonded region, whereas the structures of the anhydrous form and the hemihydrate contain two distinct regions of hydrogen bonding, as discussed above. The hydrogen-bonded region in the monohydrate is described in terms of two hydrogen-bonded sheets (Fig. 11) parallel to the $a b$-plane, with the two sheets connected by further hydrogen bonding; the hydrogen bonding involves the amine group of the side-chain, the carboxylate and ammonium groups of the amino acid head-group, and the water molecule. The ammonium group forms three $\mathrm{N}-\mathrm{H} \cdots \mathrm{O}$ hydrogen bonds as a donor, in two cases with oxygen atoms in different carboxylate groups as the acceptor (one of which is not seen in Fig. 11 as it links the two hydrogenbonded sheets) and in one case with the oxygen atom of a water molecule as the acceptor. The amine group forms one $\mathrm{N}-\mathrm{H} \cdots \mathrm{O}$ hydrogen bond as a donor with a carboxylate oxygen atom, one $\mathrm{N}-\mathrm{H} \cdots \mathrm{O}$ hydrogen bond as a donor with a water molecule, and one $\mathrm{N} \cdots \mathrm{H}-\mathrm{O}$ hydrogen bond as an acceptor with a water molecule as the donor. The water molecule forms one $\mathrm{O}-\mathrm{H} \cdots \mathrm{N}$ hydrogen bond as a donor to the aforementioned amine group and one $\mathrm{O}-\mathrm{H} \cdots \mathrm{O}$ hydrogen bond as a donor with a carboxylate oxygen atom (the latter hydrogen bond is also not seen in Fig. 11 as it links the two sheets). The water molecule also acts as an acceptor in an $\mathrm{O} \cdots \mathrm{H}-\mathrm{N}$ hydrogen bond with an ammonium group, as mentioned above.

It is interesting to note that the unit cell volume is lower for L-lysine monohydrate than for Llysine hemihydrate. The calculated densities of anhydrous L-lysine ${ }^{1}\left(1.14 \mathrm{~g} \mathrm{~cm}^{-3}\right)$ and L-lysine hemihydrate $\left(1.16 \mathrm{~g} \mathrm{~cm}^{-3}\right)$ are very similar, while the calculated density of L-lysine monohydrate $\left(1.24 \mathrm{~g} \mathrm{~cm}^{-3}\right)$ is significantly higher, reflecting the similar packing arrangements in anhydrous Llysine and L-lysine hemihydrate, and the significantly differing packing arrangement in L-lysine monohydrate. 


\section{Concluding Remarks}

In conclusion, the crystal structures of both L-lysine hemihydrate and L-lysine monohydrate have been determined directly from powder XRD data. Structure determination from powder XRD was essential due to the difficulty in preparing a single crystal of sufficient size and quality for structure determination from single-crystal XRD. The hydration/dehydration behaviour of the anhydrous and hydrated forms of L-lysine has been characterized by DVS, which further confirms the highly hygroscopic nature of anhydrous L-lysine.

From the similarity between the crystal structures of anhydrous L-lysine and L-lysine hemihydrate (Fig. 9), it is reasonable to postulate that the transformation between these solid forms should be both facile and reversible. Furthermore, we anticipate that the hydrogen-bonding arrangement involving the side-chain amine groups and water molecules in L-lysine hemihydrate (i.e., the region shaded red in Fig. 9b) is likely to be more favourable than the corresponding hydrogenbonding arrangement in anhydrous L-lysine involving only the side-chain amine groups (i.e., the region shaded red in Fig. 9a). As a consequence, it is not surprising that anhydrous L-lysine is found to have a strong propensity to undergo hydration, even at very low levels of relative humidity, as supported by our DVS results. Thus, the transformation of anhydrous L-lysine to L-lysine hemihydrate occurs reversibly and at very low values of relative humidity, reflecting the highly hygroscopic nature of anhydrous L-lysine. The transformation of L-lysine hemihydrate to L-lysine monohydrate involves substantially greater reorganization of the crystal structure. However, no mechanistic interpretations can be verified from the DVS measurements, as the formation of L-lysine monohydrate was found to occur simultaneously with a deliquescence event. Clearly, it is possible that the transformation of Llysine hemihydrate to L-lysine monohydrate involves the direct action of water in contact with the solid, present due to the partial deliquescence of the sample (for example, via dissolution and recrystallization). 


\section{ASSOCIATED CONTENT}

\section{Supporting Information}

Crystal information (cif) files for the crystal structures of L-lysine hemihydrate and L-lysine monohydrate, as well as additional Figures. This information is available free of charge via the Internet at http://pubs.acs.org/

\section{AUTHOR INFORMATION}

\section{Corresponding Author}

* E-mail: HarrisKDM@cardiff.ac.uk

\section{Notes}

The authors declare no competing financial interest.

\section{ACKNOWLEDGEMENTS}

We are grateful to Cardiff University for financial support. We thank the Ecole Nationale Supérieure de Chimie de Clermont-Ferrand for providing the opportunity for JM and EC to spend summer placements at Cardiff University, during which their contributions to this research project were carried out. 


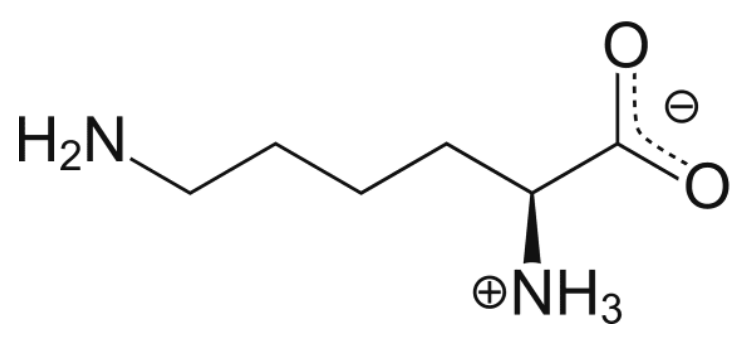

Figure 1: Molecular structure of L-lysine.

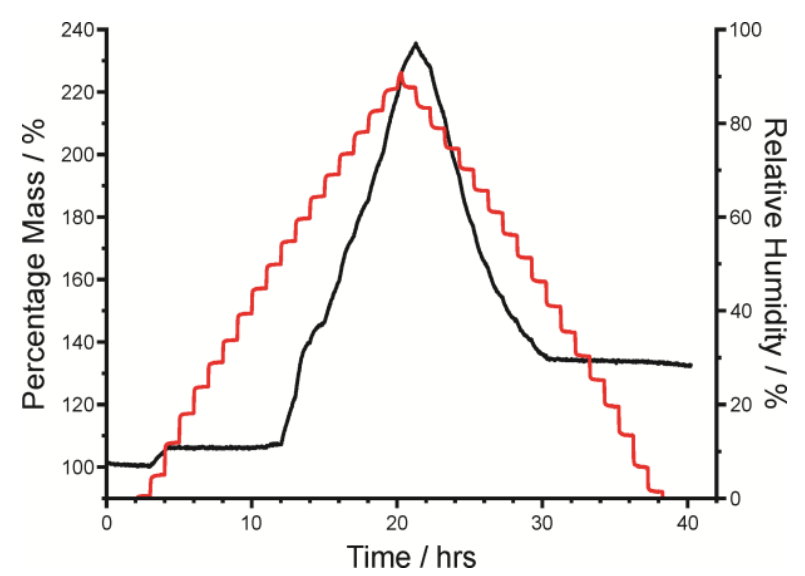

Figure 2: DVS data starting from a sample of anhydrous L-lysine (prepared by dehydration of L-lysine hemihydrate under vacuum in the presence of $\left.\mathrm{P}_{2} \mathrm{O}_{5}\right)$. The target value of relative humidity (RH) was increased from $0 \%$ to $95 \%$ in steps of $5 \%$ and then decreased back to $0 \%$ in steps of $-5 \%$; the actual value of RH in the sample environment is shown by the red line. The sample mass (black line) is expressed as a percentage of the lowest mass recorded (at $0 \% \mathrm{RH}$ ). The slight decrease in sample mass during the first ca. 2 hrs of the experiment may be attributed to loss of surface adsorbed water; mass percentage of $100 \%$ is defined as the lowest mass recorded following this initial slight loss of mass. 


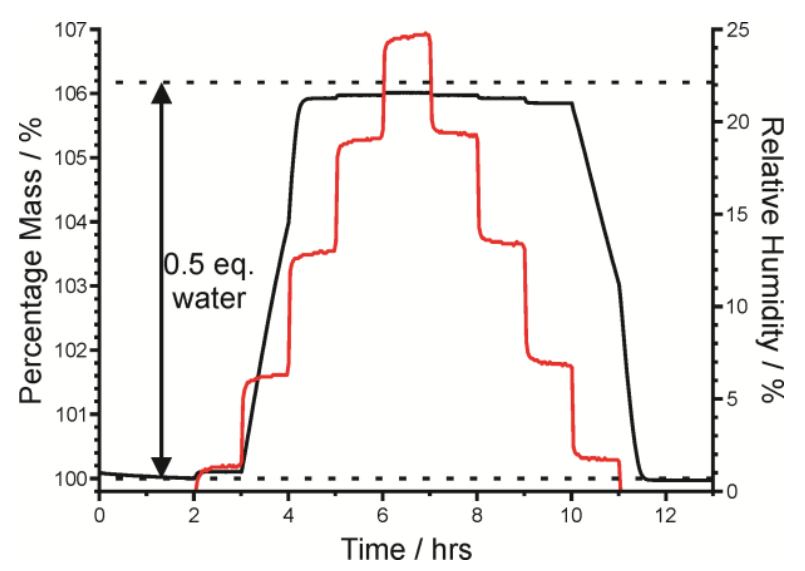

Figure 3: DVS data starting from a sample of anhydrous L-lysine (prepared by dehydration of L-lysine hemihydrate under vacuum in the presence of $\left.\mathrm{P}_{2} \mathrm{O}_{5}\right)$. The target value of relative humidity $(\mathrm{RH})$ was increased from $0 \%$ to $25 \%$ in steps of $5 \%$ and then decreased back to $0 \%$ in steps of $-5 \%$; the actual value of RH in the sample environment is shown by the red line. The sample mass (black line) is expressed as a percentage of the lowest mass recorded (at $0 \% \mathrm{RH})$. The horizontal dashed line at mass percentage $c a$. $106.2 \%$ corresponds to uptake of one half molecule of water per molecule of L-lysine. The slight decrease in sample mass during the first $c a .2 \mathrm{hrs}$ of the experiment may be attributed to loss of surface adsorbed water; mass percentage of $100 \%$ is defined as the lowest mass recorded following this initial slight loss of mass. 
a

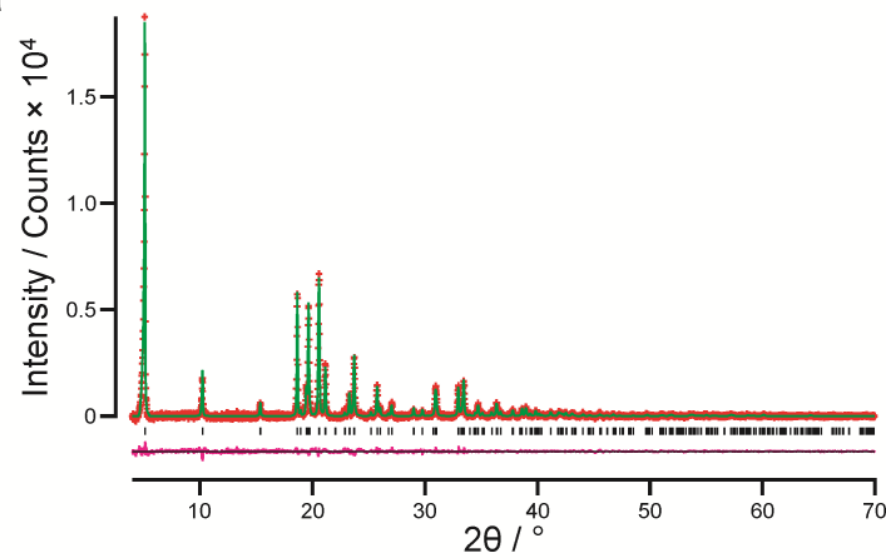

b

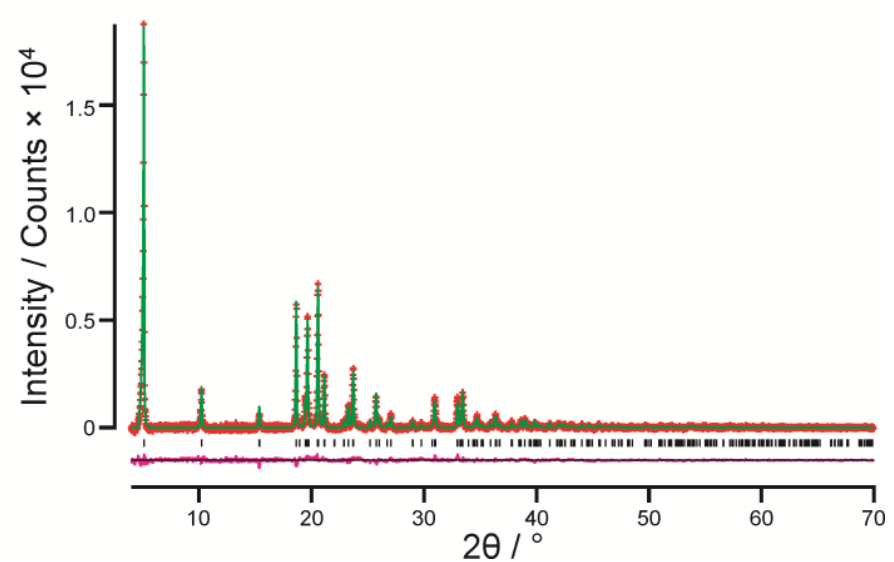

Figure 4: Results from (a) profile refinement (using the Le Bail technique) and (b) Rietveld refinement for the powder XRD pattern (following background subtraction) of L-lysine hemihydrate, showing experimental data (red + marks), calculated data (green line), difference plot (magenta line) and predicted peak positions (black tick marks). 
a

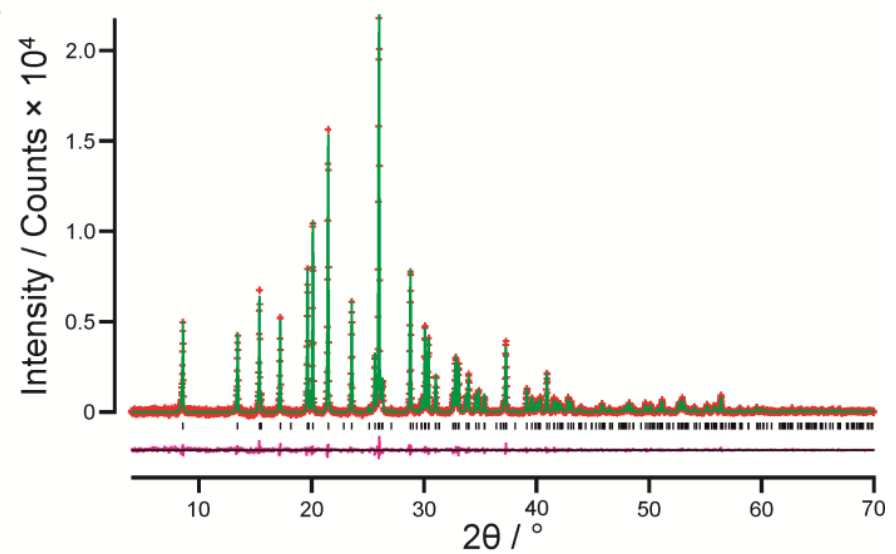

b

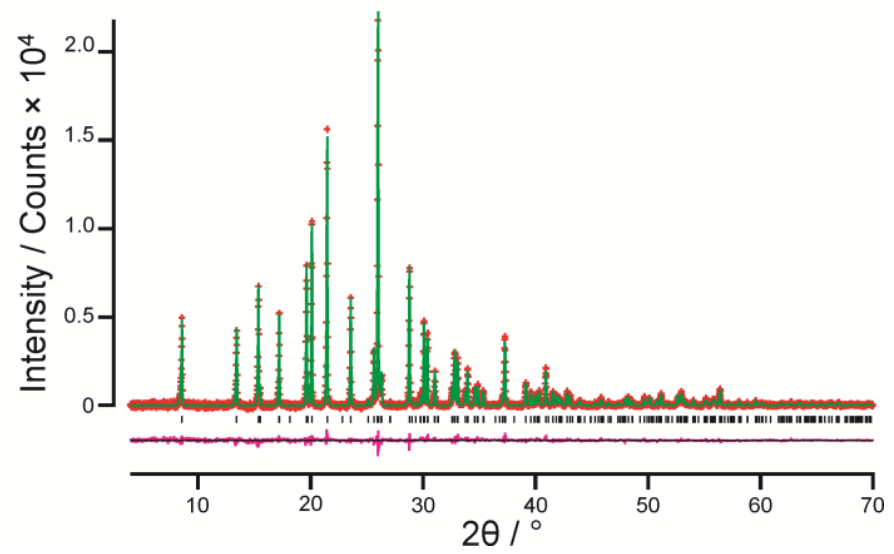

Figure 5: Results from (a) profile refinement (using the Le Bail technique) and (b) Rietveld refinement for the powder XRD pattern (following background subtraction) of L-lysine monohydrate, showing the experimental data (red + marks), calculated data (green line), difference plot (magenta line), and predicted peak positions (black tick marks).

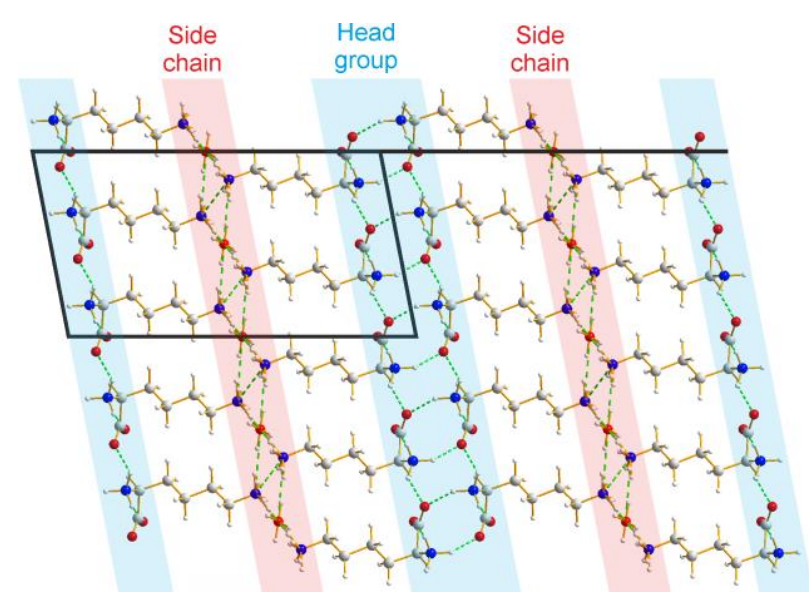

Figure 6: Crystal structure of L-lysine hemihydrate viewed along the $b$-axis. Hydrogen bonds are shown as green dashed lines. The region of hydrogen bonding involving the head-groups is shaded blue. The region of hydrogen bonding involving the side-chains and water molecules is shaded red. 


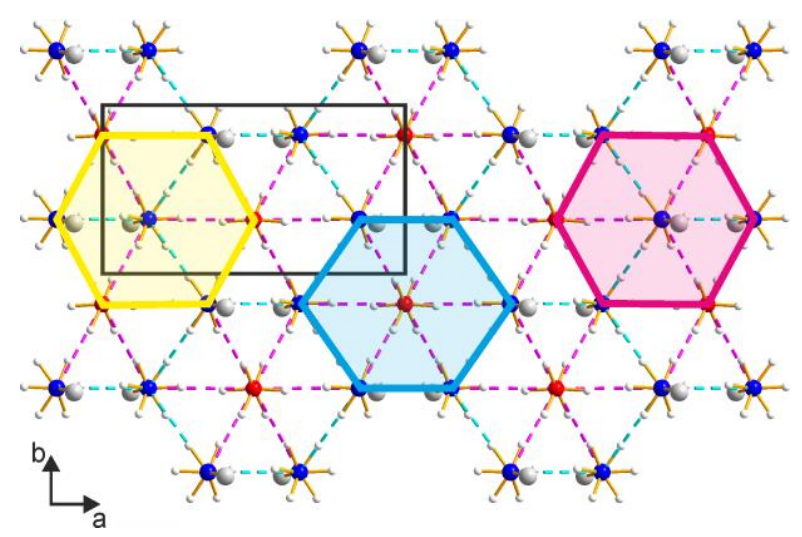

Figure 7: The disordered hydrogen-bonding arrangement in the crystal structure of L-lysine hemihydrate, involving the side-chain amine groups of L-lysine molecules and water molecules. $\mathrm{N}-\mathrm{H} \cdots \mathrm{N}$ hydrogen bonds are shown as cyan dashed lines and $\mathrm{N}-\mathrm{H} \cdots \mathrm{O}$ hydrogen bonds are shown as magenta dashed lines. The hexagon shaded in cyan illustrates the chair-type arrangement constructed from amine groups whereas the hexagons shaded in magenta and yellow illustrate the two chair-type arrangements constructed from alternating amine groups and water molecules.

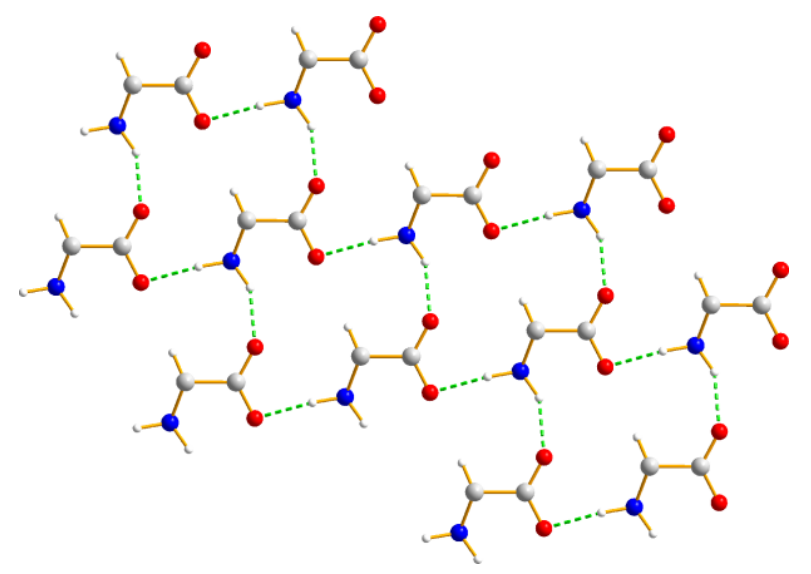

Figure 8: A single layer showing the hydrogen-bonding arrangement of the amino acid head-groups in the crystal structure of L-lysine hemihydrate. Hydrogen bonds are indicated by green dashed lines. The hydrogen-bonding arrangement is classified as $L x$ in the classification system of Görbitz et al. ${ }^{44}$ 

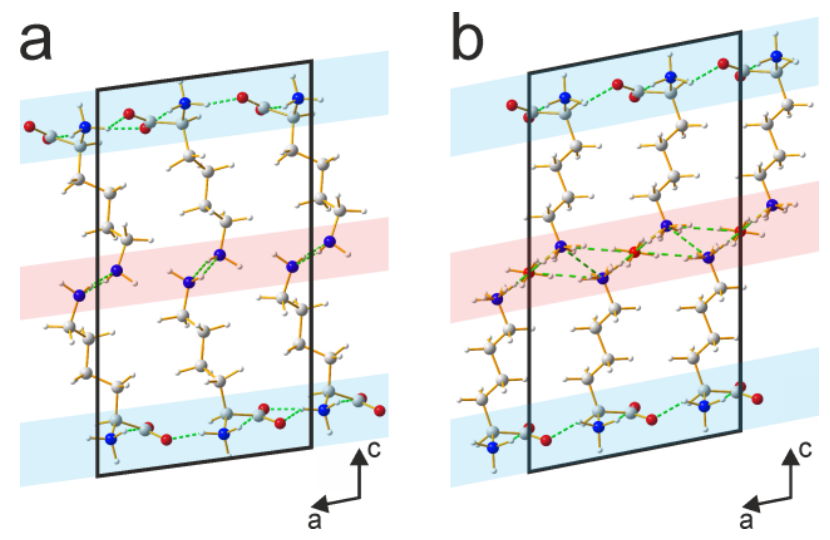

Figure 9: Comparison of the crystal structures of (a) anhydrous L-lysine ${ }^{1}$ and (b) L-lysine hemihydrate, revealing the structural similarity between these materials. The water molecules in the hemihydrate are incorporated into the region involving the side-chain amine groups of L-lysine molecules (shaded in red). The region containing the hydrogen-bonded arrangement of amino acid head-groups in each structure is shaded in blue.

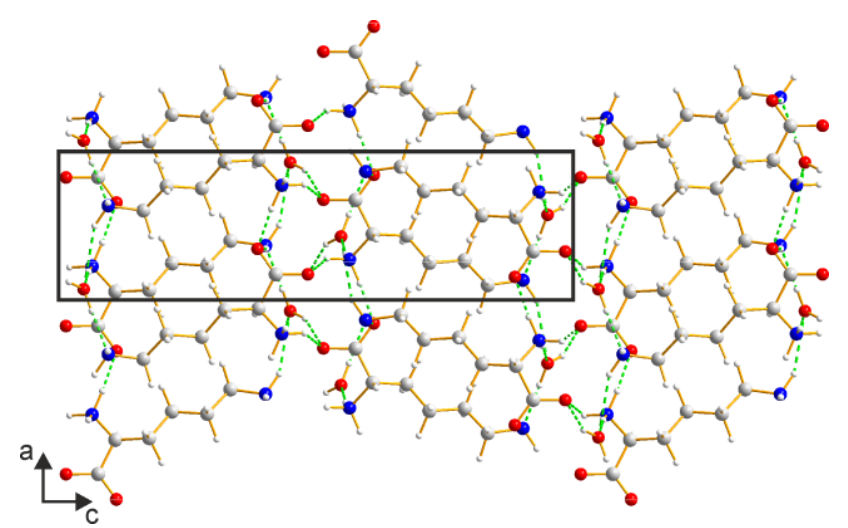

Figure 10: Crystal structure of L-lysine monohydrate viewed along the $b$-axis. Hydrogen bonds are indicated by green dashed lines.

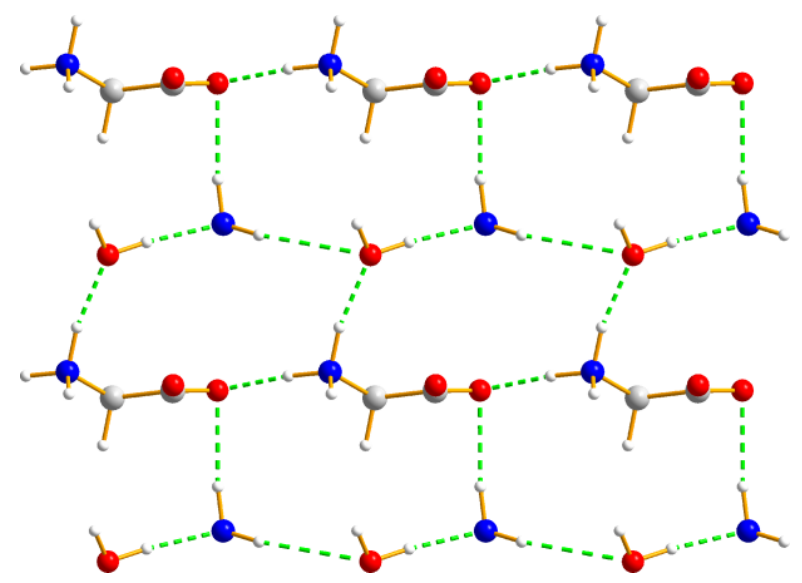

Figure 11: A single hydrogen-bonded sheet in the crystal structure of L-lysine monohydrate, viewed along the $c$-axis. Hydrogen bonds are indicated by green dashed lines. 


\section{References}

1. Williams, P. A.; Hughes, C. E.; Harris, K. D. M., L-Lysine: Exploiting Powder X-ray Diffraction to Complete the Set of Crystal Structures of the 20 Directly Encoded Proteinogenic Amino Acids. Angew. Chem. Int. Ed. 2015, 54, 3973-3977.

2. Görbitz, C. H., Crystal Structures of Amino Acids: from Bond Lengths in Glycine to Metal Complexes and High-pressure Polymorphs. Crystallogr. Rev. 2015, 21, 160-212.

3. Williams, P. A.; Hughes, C. E.; Buanz, A. B. M.; Gaisford, S.; Harris, K. D. M., Expanding the Solid-State Landscape of L-Phenylalanine: Discovery of Polymorphism and New Hydrate Phases, with Rationalization of Hydration/Dehydration Processes. J. Phys. Chem. C 2013, 117, 12136-12145.

4. Courvoisier, E.; Williams, P. A.; Lim, G. K.; Hughes, C. E.; Harris, K. D. M., The Crystal Structure of L-Arginine. Chem. Commun. 2012, 48, 2761-2763.

5. Ciunik, Z.; Glowiak, T., DL-Glutamic Acid Monohydrate, $\mathrm{C}_{5} \mathrm{H}_{9} \mathrm{NO}_{4} \cdot \mathrm{H}_{2} \mathrm{O}$. Acta Crystallogr. Sect. C 1983, 39, 1271-1273.

6. Kartha, G.; De Vries, A., Structure of Asparagine Monohydrate. Nature 1961, 192, 862-863.

7. Umadevi, K.; Anitha, K.; Sridhar, B.; Srinivasan, N.; Rajaram, R. K., L-Aspartic Acid Monohydrate. Acta Crystallogr. Sect. E 2003, 59, o1073-o1075.

8. Karle, I. L.; Karle, J., An Application of the Symbolic Addition Method to the Structure of LArginine Dihydrate. Acta Crystallogr. 1964, 17, 835-841.

9. Kingsford-Adaboh, R.; Grosche, M.; Dittrich, B.; Luger, P., DL-Arginine Monohydrate at 100 K. Acta Crystallogr. Sect. C 2000, 56, 1274-1276.

10. Padmanabhan, S.; Suresh, S.; Vijayan, M., DL-Proline Monohydrate. Acta Crystallogr. Sect. C 1995, 51, 2098-2100.

11. Janczak, J.; Luger, P., L-Proline Monohydrate at 100 K. Acta Crystallogr. Sect. C 1997, 53, 1954-1956.

12. Frey, M. N.; Lehmann, M. S.; Koetzle, T. F.; Hamilton, W. C., Precision Neutron Diffraction Structure Determination of Protein and Nucleic Acid Components. XI. Molecular Configuration and Hydrogen Bonding of Serine in the Crystalline Amino Acids L-Serine Monohydrate and DL-Serine. Acta Crystallogr. Sect. B 1973, 29, 876-884.

13. Chakraborty, S.; Bera, A. K.; Ghosh, S.; Bhattacharya, S.; Pal, A. K.; Mukhopadhyay, B. P.; Banerjee, A., Hydrogen Bonding Potentiality of Serine in More Aquated Environment: Crystal 
and Molecular Structure of Dihydrated DL-Serine, $\mathrm{C}_{3} \mathrm{H}_{7} \mathrm{NO}_{3} .2 \mathrm{H}_{2} \mathrm{O}$. Indian J. Phys., $A$ 2001, 75 , 499-501.

14. Lightfoot, P.; Tremayne, M.; Harris, K. D. M.; Bruce, P. G., Determination of a Molecular Crystal Structure by X-ray Powder Diffraction on a Conventional Laboratory Instrument. $J$. Chem. Soc. Chem. Commun. 1992, 1012-1013.

15. Harris, K. D. M.; Tremayne, M.; Lightfoot, P.; Bruce, P. G., Crystal Structure Determination from Powder Diffraction Data by Monte Carlo Methods. J. Am. Chem. Soc. 1994, 116, 35433547.

16. Chernyshev, V. V., Structure Determination from Powder Diffraction. Russ. Chem. Bull. 2001, 50, 2273-2292.

17. Tremayne, M., The Impact of Powder Diffraction on the Structural Characterization of Organic Crystalline Materials. Philos. Trans. R. Soc. A 2004, 362, 2691-2707.

18. Tsue, H.; Horiguchi, M.; Tamura, R.; Fujii, K.; Uekusa, H., Crystal Structure Solution of Organic Compounds from X-ray Powder Diffraction Data. J. Synth. Org. Chem. Jpn. 2007, 65, 1203-1212.

19. David, W. I. F.; Shankland, K., Structure Determination from Powder Diffraction Data. Acta Crystallogr. Sect. A 2008, 64, 52-64.

20. Harris, K. D. M., Powder Diffraction Crystallography of Molecular Solids. Top. Curr. Chem. 2012, 315, 133-178.

21. Guo, F.; Harris, K. D. M., Structural Understanding of a Molecular Material that is Accessed only by a Solid-state Desolvation Process: The Scope of Modern Powder X-ray Diffraction Techniques. J. Am. Chem. Soc. 2005, 127, 7314-7315.

22. Albesa-Jové, D.; Pan, Z.; Harris, K. D. M.; Uekusa, H., A Solid-state Dehydration Process Associated with a Significant Change in the Topology of Dihydrogen Phosphate Chains, Established from Powder X-ray Diffraction. Cryst. Growth Des. 2008, 8, 3641-3645.

23. Kohlbeck, F.; Hörl, E. M., Trial and Error Indexing Program for Powder Patterns of Monoclinic Substances. J. Appl. Crystallogr. 1978, 11, 60-61.

24. Visser, J. W., A Fully Automatic Program for Finding Unit Cell from Powder Data. J. Appl. Crystallogr. 1969, 2, 89-95.

25. Shirley, R., The CRYSFIRE System for Automatic Powder Indexing: User's Manual. The Lattice Press: Guildford, U.K., 1999. 
26. Le Bail, A.; Duroy, H.; Fourquet, J. L., Ab-Initio Structure Determination of LiSbWO 6 by Xray Powder Diffraction. Mater. Res. Bull. 1988, 23, 447-452.

27. Larson, A. C.; Von Dreele, R. B., General Structure Analysis System (GSAS). Los Alamos National Laboratory Report 2004, LAUR 86-748.

28. Kariuki, B. M.; Serrano-González, H.; Johnston, R. L.; Harris, K. D. M., The Application of a Genetic Algorithm for Solving Crystal Structures from Powder Diffraction Data. Chem. Phys. Lett. 1997, 280, 189-195.

29. Harris, K. D. M.; Johnston, R. L.; Kariuki, B. M., The Genetic Algorithm: Foundations and Applications in Structure Solution from Powder Diffraction Data. Acta Crystallogr. Sect. A 1998, 54, 632-645.

30. Turner, G. W.; Tedesco, E.; Harris, K. D. M.; Johnston, R. L.; Kariuki, B. M., Implementation of Lamarckian Concepts in a Genetic Algorithm for Structure Solution from Powder Diffraction Data. Chem. Phys. Lett. 2000, 321, 183-190.

31. Habershon, S.; Harris, K. D. M.; Johnston, R. L., Development of a Multipopulation Parallel Genetic Algorithm for Structure Solution from Powder Diffraction Data. J. Comput. Chem. 2003, 24, 1766-1774.

32. Harris, K. D. M.; Habershon, S.; Cheung, E. Y.; Johnston, R. L., Developments in Genetic Algorithm Techniques for Structure Solution from Powder Diffraction Data. Z. Kristallogr. 2004, 219, 838-846.

33. Tedesco, E.; Turner, G. W.; Harris, K. D. M.; Johnston, R. L.; Kariuki, B. M., Structure Determination of an Oligopeptide Directly from Powder Diffraction Data. Angew. Chem. Int. Ed. 2000, 39, 4488-4491.

34. Albesa-Jové, D.; Kariuki, B. M.; Kitchin, S. J.; Grice, L.; Cheung, E. Y.; Harris, K. D. M., Challenges in Direct-space Structure Determination from Powder Diffraction Data: A Molecular Material with Four Independent Molecules in the Asymmetric Unit. ChemPhysChem 2004, 5, 414-418.

35. Pan, Z.; Xu, M.; Cheung, E. Y.; Harris, K. D. M.; Constable, E. C.; Housecroft, C. E., Understanding the Structural Properties of a Dendrimeric Material Directly from Powder X-ray Diffraction Data. J. Phys. Chem. B 2006, 110, 11620-11623.

36. Guo, F.; Martí-Rujas, J.; Pan, Z.; Hughes, C. E.; Harris, K. D. M., Direct Structural Understanding of a Topochemical Solid State Photopolymerization Reaction. J. Phys. Chem. C 2008, 112, 19793-19796. 
37. Fujii, K.; Garay, A. L.; Hill, J.; Sbircea, E.; Pan, Z.; Xu, M.; Apperley, D. C.; James, S. L.; Harris, K. D. M., Direct Structure Elucidation by Powder X-ray Diffraction of a Metal-Organic Framework Material Prepared by Solvent-Free Grinding. Chem. Commun. 2010, 46, 75727574.

38. Fujii, K.; Young, M. T.; Harris, K. D. M., Exploiting Powder X-ray Diffraction for Direct Structure Determination in Structural Biology: The P2X4 Receptor Trafficking Motif YEQGL. J. Struct. Biol. 2011, 174, 461-467.

39. Martí-Rujas, J.; Meazza, L.; Lim, G. K.; Terraneo, G.; Pilati, T.; Harris, K. D. M.; Metrangolo, P.; Resnati, G., An Adaptable and Dynamically Porous Organic Salt Traps Unique Tetrahalide Dianions. Angew. Chem. Int. Ed. 2013, 52, 13444-13448.

40. Rietveld, H. M., A Profile Refinement Method for Nuclear and Magnetic Structures. J. Appl. Crystallogr. 1969, 2, 65-71.

41. Standard bond-length and bond-angle restraints were obtained from the mean values of similar structures deposited in the Cambridge Structural Database, as determined using the program Mogul (version 1.7) for bonds between non-hydrogen atoms. Bond-length restraints for bonds involving hydrogen atoms were taken from: Allen, F. H.; Kennard, O.; Watson, D. G.; Brammer, L.; Orpen, A. G.; Taylor, R., Tables of Bond Lengths Determined by X-ray and Neutron Diffraction. Part 1. Bond Lengths in Organic Compounds. J. Chem. Soc. Perkin Trans. 2 1987, S1-S19.

42. March, A., Mathematical Theory on Regulation According to the Particle Shape in Affine Deformation. Z. Kristallogr. 1932, 81, 285-297.

43. Dollase, W. A., Correction of Intensities for Preferred Orientation in Powder Diffractometry Application of the March Model. J. Appl. Crystallogr. 1986, 19, 267-272.

44. Görbitz, C. H.; Vestli, K.; Orlando, R., A Solution to the Observed $Z^{\prime}=2$ Preference in the Crystal Structures of Hydrophobic Amino Acids. Acta Crystallogr. Sect. B 2009, 65, 393-400. 
For table of contents only:

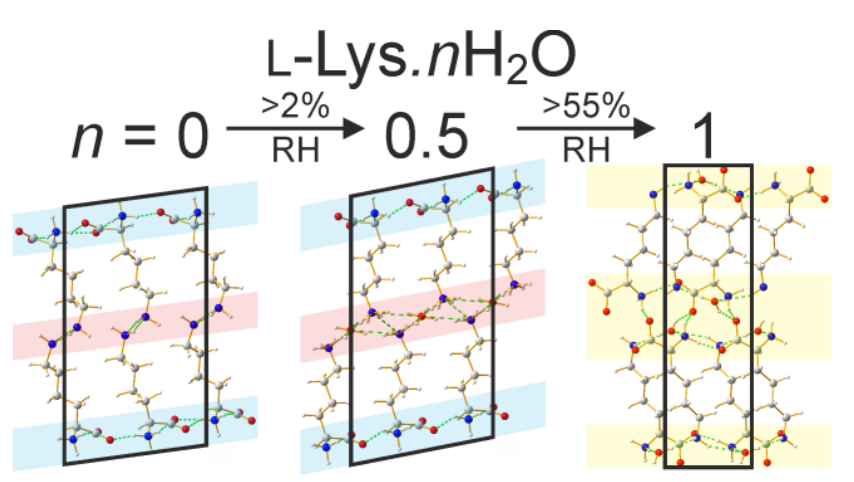

